\title{
Randomly dilute spin models with cubic symmetry.
}

\author{
Pasquale Calabrese $^{1}$, Andrea Pelissetto ${ }^{2}$, Ettore Vicari ${ }^{3}$ \\ ${ }^{1}$ Scuola Normale Superiore and I.N.F.N., Piazza dei Cavalieri 7, I-56126 Pisa, Italy. \\ 2 Dipartimento di Fisica dell'Università di Roma I and I.N.F.N., I-00185 Roma, Italy. \\ 3 Dipartimento di Fisica dell'Università and I.N.F.N., Via Buonarroti 2, I-56127 Pisa, Italy. \\ e-mail: calabres@df.unipi.it, Andrea.Pelissetto@roma1.infn.it, vicari@df .unipi.it.
}

(October 31, 2018)

\begin{abstract}
We study the combined effect of cubic anisotropy and quenched uncorrelated impurities on multicomponent spin models. For this purpose, we consider the field-theoretical approach based on the Ginzburg-Landau-Wilson $\varphi^{4}$ Hamiltonian with cubic-symmetric quartic interactions and quenched randomness coupled to the local energy density. We compute the renormalization-group functions to six loops in the fixed-dimension $(d=3)$ perturbative scheme. The analysis of such high-order series provides an accurate description of the renormalization-group flow. The results are also used to determine the critical behavior of three-dimensional antiferromagnetic three- and four-state Potts models in the presence of quenched impurities.
\end{abstract}

PACS Numbers: 75.10.Nr, 05.70.Jk, 64.60.Ak, 75.40.-s 


\section{INTRODUCTION AND MAIN RESULTS.}

The critical behavior of systems with quenched disorder is of considerable theoretical and experimental interest. A typical example is obtained by mixing an (anti)-ferromagnetic material with a nonmagnetic one, obtaining the so-called randomly dilute magnets. They are usually described by using the Heisenberg Hamiltonian with a random-exchange term

$$
\mathcal{H}_{H, r}=-J \sum_{<x y>} \rho_{x} \rho_{y} \vec{s}_{x} \cdot \vec{s}_{y}
$$

where $s_{x, i}$ are $M$-component spins and $\rho_{x}$ are uncorrelated random variables, which are equal to one with probability $p$ (the spin concentration) and zero with probability $1-p$ (the impurity concentration). The pure system corresponds to $p=1$. The critical behavior of these systems is well established, both theoretically and experimentally, see, e.g., Refs. [1-5] and references therein. In particular, a new random Ising universality class describes the critical behavior of the random Ising model (RIM) above the percolation threshold of the magnetic atoms.

The $\mathrm{O}(M)$-symmetric Hamiltonian (1.1) is a rather simplified model for real magnets. In particular, it does not take into account the presence of nonrotationally invariant interactions that have only the reduced symmetry of the lattice and that are due, e.g., to the spin-lattice coupling and to dipole-dipole interactions. In this case, a more realistic Hamiltonian is

$$
\mathcal{H}_{\mathrm{H}, \mathrm{c}}=-J \sum_{<x y>} \vec{s}_{x} \cdot \vec{s}_{y}+a \sum_{x} \sum_{i} s_{x, i}^{4}
$$

where $a$ is the anisotropy coupling. For many materials $a / J$ is relatively small and thus one usually neglects these additional interactions. However, this is fully justified only if they are irrelevant in the renormalization-group ( $R G)$ sense. This issue may be investigated by considering the cubic-symmetric $\varphi^{4}$ Hamiltonian [2]

$$
\mathcal{H}_{c}=\int d^{d} x\left\{\frac{1}{2}\left(\partial_{\mu} \phi(x)\right)^{2}+\frac{1}{2} r \phi(x)^{2}+\frac{1}{4 !} v\left[\phi(x)^{2}\right]^{2}+\frac{1}{4 !} w \sum_{i=1}^{M} \phi_{i}(x)^{4}\right\}
$$

where $\phi(x)$ is an $M$-component field and $r \propto T-T_{c}$. Analyses of high-order perturbative expansions (see, e.g., Refs. [6-10]) show that the cubic-symmetric quartic $w$-interaction is relevant for $M \geq 3$, and in particular, for the physically relevant case $M=3$. In this case, the nature of the transition depends on the sign of the coupling $w$ : if $w>0$, the critical behavior is described by a new fixed point with reduced cubic symmetry, while, for $w<0$, the RG flow runs away to infinity, and the corresponding system is expected to undergo a weak first-order transition. In the two-component case, the $\mathrm{O}(2)$-symmetric fixed point is stable with respect to the $w$-perturbation, and thus, if the transition is continuous, it belongs to the XY universality class.

It is of interest to study the effect of quenched disorder on cubic magnets. As discussed in Ref. [12], the critical behavior of these materials should be described by the effective Hamiltonian

$$
\mathcal{H}_{H, r c}=-J \sum_{<x y>} \rho_{x} \rho_{y} \vec{s}_{x} \cdot \vec{s}_{y}+a \sum_{x} \sum_{i} s_{x, i}^{4}+\sum_{x} \sum_{i j} D_{x, i j} s_{x, i} s_{x, j}
$$


where, beside the random-exchange term, a random-anisotropy term is present. Here the anisotropy term $D_{x, i j}$ is a random quantity that is traceless and has zero average. Note that, for small anisotropy and weak disorder, this additional term should be smaller than the other ones, being, loosely speaking, proportional to the product of $(1-p)$ and $a$, i.e., it is a second-order perturbation of the Heisenberg Hamiltonian. This argument is not fully justified at criticality, since, if random anisotropy is relevant, it will eventually change the critical behavior. Nonetheless, we expect-as we shall see, experiments confirm this assumption - a large preasymptotic region in which such term can be neglected. For this reason in this paper we will not consider the random-anisotropy term and we shall discuss the critical behavior of the model with Hamiltonian (1.4) with $D_{x, i j}=0$.

If only the random-exchange term is present, i.e. $D_{x, i j}=0$, the critical behavior of the model (1.4) can by studied using the field-theoretical Hamiltonian

$$
\mathcal{H}_{r c}=\int d^{d} x\left\{\frac{1}{2}\left(\partial_{\mu} \phi(x)\right)^{2}+\frac{1}{2} r \phi(x)^{2}+\frac{1}{2} \psi(x) \phi(x)^{2}+\frac{1}{4 !} v\left[\phi(x)^{2}\right]^{2}+\frac{1}{4 !} w \sum_{i=1}^{M} \phi_{i}(x)^{4}\right\},
$$

where $\psi(x)$ is a spatially uncorrelated random field with Gaussian distribution coupled to the local energy density. Using the standard replica trick, one obtains the Hamiltonian $[13,14]$

$$
\mathcal{H}_{e}=\int d^{d} x\left\{\sum_{i, a} \frac{1}{2}\left[\left(\partial_{\mu} \phi_{a, i}\right)^{2}+r \phi_{a, i}^{2}\right]+\sum_{i j, a b} \frac{1}{4 !}\left(u+v \delta_{i j}+w \delta_{i j} \delta_{a b}\right) \phi_{a, i}^{2} \phi_{b, j}^{2}\right\},
$$

where $a, b=1, \ldots M$ and $i, j=1, \ldots N$. The original system, i.e. the randomly dilute $M$ component cubic model, is recovered in the $N \rightarrow 0$ limit. [15] The coupling $u$ is negative, being proportional to minus the variance of the quenched disorder.

The study of the effective Hamiltonian $\mathcal{H}_{e}$ in the limit $N \rightarrow 0$ provides also information on the critical behavior of the randomly dilute antiferromagnetic $q$-state Potts model for $q=2$ and 3 , with Hamiltonian

$$
\mathcal{H}_{d q}=J \sum_{<x y>} \rho_{x} \rho_{y} \delta_{s_{x}, s_{y}},
$$

where $J>0, s_{x}=1, \ldots, q$, and $\rho_{x}$ are uncorrelated random variables. Indeed, as argued in Refs. [20,21] using RG arguments, the critical behavior of the antiferromagnetic three- and four-state Potts models on a cubic lattice should be described by the cubic Hamiltonian $\mathcal{H}_{c}$ with $M=2$ and $M=3$ respectively and with $w<0$. The same correspondence holds in the random case. The randomly dilute three- and four-state models are respectively related to the two- and three-component model with Hamiltonian (1.6) in the limit $N \rightarrow 0$ and for $w<0$.

Since disorder is coupled to the local energy density, one can use the Harris criterion [11] to predict the critical behavior of the model. It states that the addition of impurities to a system that undergoes a second-order phase transition does not change the critical behavior if the specific-heat critical exponent $\alpha_{\text {pure }}$ of the pure system is negative. If $\alpha_{\text {pure }}$ is positive, the transition is altered. This occurs in the Ising case $(M=1)$, where the addition of impurities leads to a new random Ising universality class (RIM). In pure $M$-component 
cubic models $(M>1)$ the specific-heat exponent $\alpha_{\text {pure }}$ is negative; therefore, according to the Harris criterion, the pure fixed point is stable against disorder. Nonetheless, disorder may still have physical consequences. For instance, it may give rise to new fixed points or change the attraction domain of the pure stable fixed point. Systems that are outside the attraction domain of the fixed point in the absence of disorder, and therefore show a fluctuation-driven first-order transition, may undergo a second-order transition in the presence of disorder. Such phenomenon, usually referred to as softening, is well-understood in two-dimensional random-exchange models in which disorder is coupled to the local energy density. Indeed, it was argued in Ref. [16], and later put on a rigorous basis $[17,18]$, that in two dimensions thermal first-order transitions become continuous in the presence of quenched disorder coupled to the local energy density. For the cubic model (1.3) for $v<0$, it was shown [19] that such a softening persists in $2+\varepsilon$ dimensions, while it is absent near four dimensions, see the $\epsilon$-expansion analysis of Sec. III. Thus, it is interesting to address this issue in three dimensions, where the analysis of Ref. [16] shows that the occurrence of softening may depend on nonuniversal features of the model.

In order to study the RG flow of the effective Hamiltonian $\mathcal{H}_{e}$, we consider the fixeddimension perturbative method in $d=3$ and compute the RG functions perturbatively to six loops. The analysis of such series allows us to determine the RG flow. We briefly anticipate the main results of our analysis. The stability of the stable fixed points of the pure theory predicted by the Harris criterion is confirmed. The region $v<0$ for any $M$ and the region $w<0$ for $M \geq 3$ is outside the attraction domain of the stable fixed point for all physical values $u<0$. Moreover, for any $M$ there exists a fixed point in the RIM universality class, which is weakly unstable (i.e. with a very small crossover exponent) and may give rise to observable crossover effects in physical systems. We do not find fixed points in the region $v<0$ for any $M$ and in the region $w<0$ for $M \geq 3$. Therefore, no softening is expected, at least for sufficiently low impurity concentration to justify the field-theoretical approach. As for the three-state random Potts model, it is expected to have an XY transition as in the pure case [22] or a first-order transition, depending on the value of the effective negative coupling $w$. The four-state random antiferromagnetic Potts model is expected to undergo a weak first-order transition.

We predict that cubic magnets with small positive anisotropy have a critical behavior controlled by the pure cubic fixed point, which has critical exponents very close to the Heisenberg ones. Therefore, experiments should effectively observe the standard $\mathrm{O}(3)$ critical exponents. This is in good agreement with the experiments that observe in most of the cases $\mathrm{O}(3)$ behavior with good accuracy, see, e.g., Refs. [4,23]. On the other hand, systems that tend to magnetize along the cubic axes, should show a first-order transition, as in the pure case. Experimentally, such a transition has never been observed, probably because of the smallness of the cubic anisotropy: due to the very small crossover exponent, $\phi \approx 0.01$, see, e.g., Refs. $[7,4]$, the cubic breaking can be observed only very close to the critical point, i.e. for $\left|T-T_{c}\right| / T_{c} \ll 10^{-4}$, which is the limit of most of the experiments. As already observed in Ref. [24], a posteriori, the experimental results also confirm the validy of neglecting the random-anisotropy term in Eq. (1.4) in the experimentally relevant range of parameters. Indeed, its presence would give rise to a crossover to a first-order transition in all cases [12]. The associated crossover exponent is $\phi=2 \phi_{H}-2+\alpha_{H} \approx 0.39$ where $\phi_{H}$ and $\alpha_{H}$ are the quadratic-anisotropy and specific-heat exponents for the Heisenberg model. Such an 

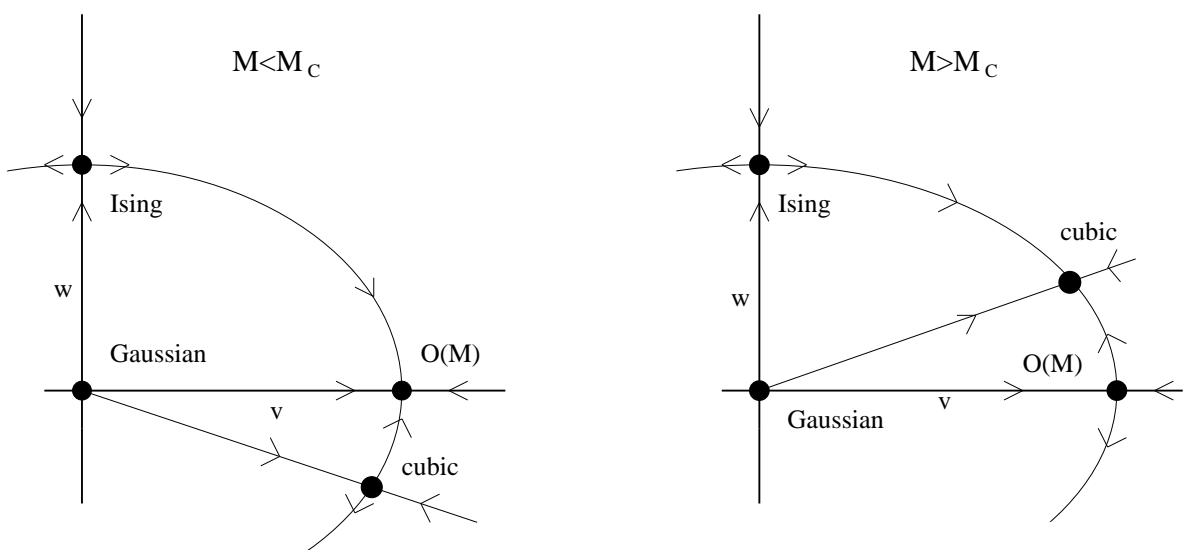

FIG. 1. RG flow in the plane $u=0$ for $M<M_{c}$ and $M>M_{c}$.

exponent is sizable and thus one should have been able to observe the random anisotropy if it were not very small.

The paper is organized as follows. In Sec. II we discuss some general properties of the RG flow in three dimensions. Sec. III analyzes the RG flow near four dimensions. In Sec. IV we present the computation and the analysis of the fixed-dimension pertubative expansion to six loops. In the Appendix we compute the differences between the three-component cubic and Heisenberg critical exponents, by a reanalysis of the fixed-dimension six-loop expansion of Ref. [7] for the cubic Hamiltonian $\mathcal{H}_{c}$.

\section{GENERAL CONSIDERATIONS ON THE RG FLOW}

In this section we discuss some properties of the RG flow of the Hamiltonian (1.6) for $N \rightarrow 0$, using general arguments and known results holding for the special cases in which one of the quartic couplings vanishes.

The RG flow in the plane $u=0$ is that of the cubic Hamiltonian (1.3) (see, e.g., Ref. [4] for a recent review of results). Indeed, for $u=0$ the Hamiltonian (1.6) describes $N$ decoupled $M$-component cubic-symmetric models. Therefore, in the plane $u=0$ there are four fixed points [2]: the trivial Gaussian one, the Ising one in which the $M$ components of the field decouple, the $\mathrm{O}(M)$-symmetric and the cubic fixed point. The Gaussian fixed point is always unstable, and so is the Ising fixed point for any number of components $M[2,25]$. On the other hand, the stability properties of the $\mathrm{O}(M)$-symmetric and of the cubic fixed point depend on $M$. For sufficiently small values of $M, M<M_{c}$, the $\mathrm{O}(M)$-symmetric fixed point is stable and the cubic one is unstable. For $M>M_{c}$, the opposite is true: the RG flow is driven towards the cubic fixed point. Figure 1 sketches the flow diagram in the two cases $M<M_{c}$ and $M>M_{c}$. High-order perturbative computations in the $\epsilon$-expansion and in the fixed-dimension field-theoretical frameworks show that $2<M_{c}<3$; more precisely, $M_{c} \approx 2.9$ [6-8]. This means that the critical behavior of the two-component cubic model is described by the $\mathrm{O}(2)$-symmetric fixed point and therefore belongs to the XY universality 
class. If $M>M_{c}$, the cubic anisotropy is relevant and therefore the critical behavior of the system is not described by the Heisenberg isotropic Hamiltonian.

In the three-component case the cubic critical exponents $\nu_{c}, \eta_{c}$ differ very little from those of the Heisenberg universality class. Indeed, the analysis of the six-loop fixed-dimension expansions of Ref. [7] reported in the Appendix provides the following estimates for their differences

$$
\nu_{c}-\nu_{H}=-0.0003(3), \quad \eta_{c}-\eta_{H}=-0.0001(1), \quad \gamma_{c}-\gamma_{H}=-0.0005(7) .
$$

Note that these differences are much smaller than the typical experimental errors, see, e.g., Ref. [4] for a list of experimental results, so that, at present, cubic effects are experimentally negligible. Using the accurate estimates of Ref. [26] for the Heisenberg exponents and Eq. (2.1), one obtains

$$
\nu_{c}=0.7109(6), \quad \eta_{c}=0.0374(5), \quad \gamma_{c}=1.3955(12),
$$

which are consistent with, but much more precise than, the results obtained from a direct analysis, see, e.g., Refs. [7,6].

The stability of the pure fixed points against the $u$-perturbation can be inferred by using general arguments $[11,25,2]$. Since the $u$-interaction is the sum of the products of the energy operators of the different cubic $M$-component models, the crossover exponent associated with $u$ is given by the specific-heat critical exponent $\alpha$ independently of $N$, and thus also for $N \rightarrow 0$. Therefore, the pure stable fixed point is stable with respect to random dilution for any $M \geq 2$, since the specific-heat exponent is always negative. For example, for $M=2$, where the stable fixed point is the $\mathrm{O}(2)$-symmetric one, we have [27] $\alpha_{\mathrm{XY}}=-0.0146(8)$; for $M=3$, where the stable fixed point is the cubic one, $\alpha_{c}=-0.133(2)$ using Eq. (2.2).

For $v=0$ the Hamiltonian (1.6) describes an $M N$-component model with cubic anisotropy. The RG flow for $N \rightarrow 0$ is shown in Fig. 2. It is characterized by the presence of two stable fixed points. The one for $u>0, w=0$ is in the self-avoiding walk (SAW) universality class, but it is irrelevant for our problem, since it is unreachable from the physical region $u<0$. The other one belongs to the region $u<0, w>0$ and it is in the RIM universality class. See, e.g., Refs. [28-32] for recent studies of the critical properties of RIM.

In the case $w=0$, the Hamiltonian (1.6) describes $N$ coupled $M$-vector models, and it is also called $M N$ model [2]. See, e.g., Ref. [4] for a recent review of results. The RG flow for $M \geq 2$ and $N \rightarrow 0$ is shown in Fig. 2. Again, the flow is characterized by two stable fixed points: the SAW and the $\mathrm{O}(M)$-symmetric ones.

For $M=2$ and generic $N$ the Hamiltonian (1.6) is invariant under the transformation

$$
\begin{aligned}
& \left(\phi_{1, i}, \phi_{2, i}\right) \longrightarrow \frac{1}{\sqrt{2}}\left(\phi_{1, i}+\phi_{2, i}, \phi_{1, i}-\phi_{2, i}\right), \\
& \left(u_{0}, v_{0}, w_{0}\right) \longrightarrow\left(u_{0}, v_{0}+\frac{3}{2} w_{0},-w_{0}\right) .
\end{aligned}
$$

For $N=0$ this transformation maps the Ising fixed point into the cubic one, and the RIM fixed point into a new one belonging to the region with $u<0, v>0, w<0$. Of course, corresponding fixed points describe the same critical behavior.

In conclusion, the above-reported considerations show the presence of at least seven fixed points for $M \geq 3$ and eight for $M=2$ using the above-mentioned symmetry. Of 

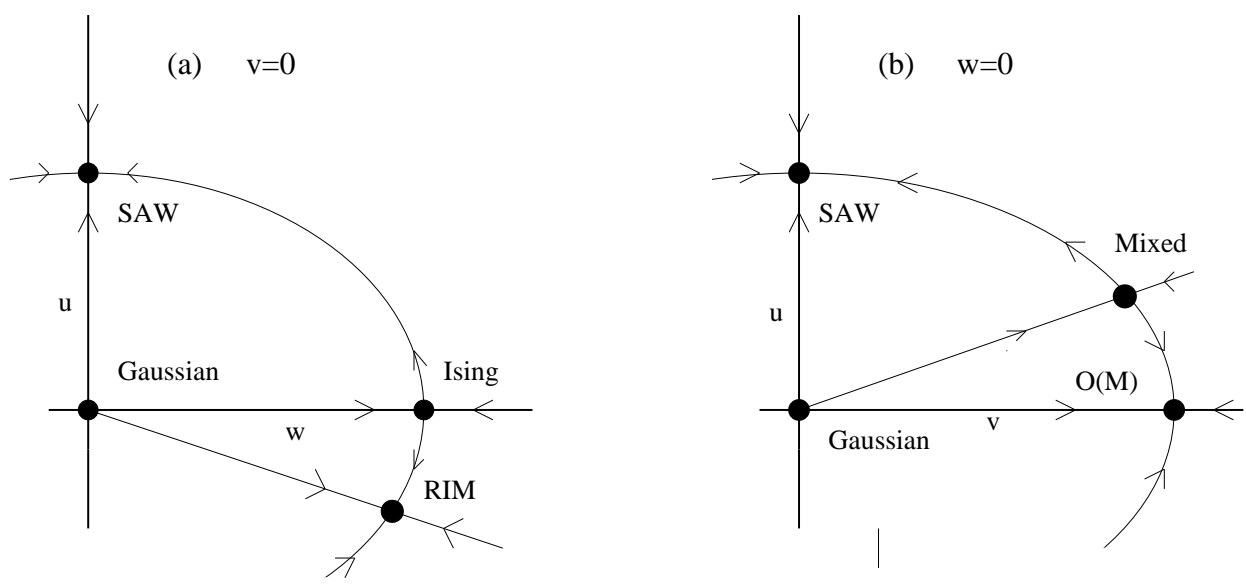

FIG. 2. RG flow in the planes $v=0$ and $w=0$ for $N=0$.

course, other fixed points may lie outside the planes $u=0, v=0, w=0$. Thus, a more general analysis for generic values of the quartic couplings is necessary in order to obtain a satisfactory knowledge of the RG flow. In particular, this would allow us to investigate if random dilution may cause a softening of the first-order transition predicted for pure systems that are outside the attraction domain of the stable fixed point. For example, systems with $v<0$ might have a continuous transition if there exists a fixed point in the region $u<0$, $v<0$, while, for systems with $M \geq 3$ and $w<0$, softening to a continuous transition requires a fixed point in the region $u<0, w<0$.

\section{THE RG FLOW NEAR FOUR DIMENSIONS}

The RG flow of the randomly dilute cubic-symmetric model can be investigated near four dimensions using the first nontrivial terms of the expansion in powers of $\epsilon \equiv 4-d$. Using the results reported in Refs. [13,33,9], one may easily obtain the results of Table I, where the location of the fixed points and the eigenvalues of the corresponding stability matrix are reported to leading order.

Note that the $O(\sqrt{\epsilon})$ fixed points emerge at two-loop level, and are related to the degeneracy of the one-loop $\beta$-functions [34]. For $M=2$ the fixed points IV, VII, VIII can be mapped respectively into the fixed points VI, IX, X using the symmetry (2.3).

The physically relevant fixed points are those that can be reached from the region $u<0$, i.e. the fixed points I (Gaussian), II $(\mathrm{O}(M))$, IV (Ising), VI (cubic), VII (RIM) for all values of $M \geq 2$, and $\mathrm{V}$ (mixed) and IX for $M \leq 4$ (note that in three dimensions the mixed fixed point $\mathrm{V}$ lies in the $u>0$ region for all $M \geq 2$, as shown in the preceding section). Concerning their stability properties, we note that all fixed points in the region $u \leq 0$, except the $O(M)$ and cubic one, are unstable for any $M$. For $M<M_{c}=4-2 \epsilon+O\left(\epsilon^{2}\right)$ ( $M_{c} \approx 2.9$ in three dimensions) the stable fixed point is the $\mathrm{O}(M)$ one, while for $M>M_{c}$ the stable fixed point is the cubic one. Note that the derivation of $\omega_{1}$ for the fixed point VII (RIM) requires a three-loop calculation — we used here the results of Ref. [33].

In conclusion, near four dimensions the critical behavior is not changed by the addition of random impurities for any $M \geq 2$. Moreover, as already observed in Ref. [19] (although 
TABLE I. Fixed points of the Hamiltonian (1.6) near four dimensions. We report the leading nontrivial contribution of the expansion in powers of $\epsilon$. Note that $K_{d}=(4 \pi)^{d} \Gamma(d / 2) / 2$.

\begin{tabular}{cccccl}
\hline \hline & & $u / K_{d}$ & $v / K_{d}$ & $w / K_{d}$ & \multicolumn{1}{c}{ stability eigenvalues } \\
\hline \hline I & Gaussian & 0 & 0 & 0 & $\omega_{u}=-\epsilon, \omega_{v}=-\epsilon, \omega_{w}=-\epsilon$ \\
II & O $(M)$ & 0 & $\frac{6}{M+8} \epsilon$ & 0 & $\omega_{u}=\frac{4-M}{M+8} \epsilon, \omega_{v}=\epsilon, \omega_{w}=\frac{4-M}{M+8} \epsilon$ \\
III & SAW & $\frac{3}{4} \epsilon$ & 0 & 0 & $\omega_{u}=\epsilon, \omega_{v}=\epsilon / 2, \omega_{w}=\epsilon / 2$ \\
IV & Ising & 0 & 0 & $\frac{2}{3} \epsilon$ & $\omega_{u}=-\epsilon / 3, \omega_{v}=\epsilon, \omega_{w}=-\epsilon / 3$ \\
V & Mixed & $\frac{M-4}{M-1} \frac{3}{8} \epsilon$ & $\frac{3}{2(M-1)} \epsilon$ & 0 & $\omega_{1}=\epsilon, \omega_{2}=\frac{4-M}{3(M+1)} \epsilon, \omega_{w}=\frac{4-M}{3(M+1)} \epsilon$ \\
VI & Cubic & 0 & $\frac{2}{M} \epsilon$ & $\frac{2(M-4)}{3 M} \epsilon$ & $\omega_{u}=\frac{M-4}{3 M} \epsilon, \omega_{2}=\epsilon, \omega_{3}=\frac{M-4}{3 M} \epsilon$ \\
VII & RIM & $-2 \sqrt{\frac{27}{106}} \sqrt{\epsilon}$ & 0 & $2 \sqrt{\frac{24}{53}} \sqrt{\epsilon}$ & $\omega_{1}=2 \epsilon, \omega_{v}=-\sqrt{\frac{24}{53}} \sqrt{\epsilon}, \omega_{3}=\sqrt{\frac{24}{53}} \sqrt{\epsilon}$ \\
VIII & \multicolumn{7}{c}{${ }^{\frac{27}{106}} \sqrt{\epsilon}$} & 0 & $-2 \sqrt{\frac{24}{53}} \sqrt{\epsilon}$ & $\omega_{1}=O(\epsilon), \omega_{v}=-\sqrt{\frac{24}{53}} \sqrt{\epsilon}, \omega_{3}=\sqrt{\frac{24}{53}} \sqrt{\epsilon}$ \\
IX & $(M>2)$ & $\frac{M-4}{4(M-2)} \epsilon$ & $\frac{1}{(M-2)} \epsilon$ & $\frac{M-4}{3(M-2)} \epsilon$ & $\omega_{1}=\epsilon, \omega_{2}=\frac{4-M}{6(M-2)} \epsilon, \omega_{3}=\frac{M-4}{6(M-2)} \epsilon$ \\
IX, X & $(M=2)$ & $\mp 2 \sqrt{\frac{27}{106}} \sqrt{\epsilon}$ & $\pm 2 \sqrt{\frac{54}{53}} \sqrt{\epsilon}$ & $\mp 2 \sqrt{\frac{24}{53}} \sqrt{\epsilon}$ \\
\hline \hline
\end{tabular}

that analysis missed the $O(\sqrt{\epsilon})$ fixed points), there is no softening of the transition for pure systems that are outside the attraction domain of the stable fixed point (in particular, for $v<0$ and for $w<0$ in the case $M \geq M_{c}$ ).

Even though the $\epsilon$ expansion provides useful indications of the RG flow in lower dimensions, the validity of the extrapolation to $\epsilon=1$ of the results obtained near four dimensions is not guaranteed, even at a qualitative level. Relevant features concerning the location and the stability of the fixed points can drastically change approaching three dimensions. Moreover, new fixed points, which are not found in $\epsilon$-expansion analyses, may appear in three dimensions. For example, this occurs in the physically interesting cases of the $\varphi^{4}$ Hamiltonian describing the critical behavior of frustrated spin models with noncollinear order, see. e.g., Refs. [35,4], and the Ginzburg-Landau model of superconductors, where a complex scalar field couples to a gauge field, see, e.g., Ref. [36].

\section{ANALYSIS OF THE SIX-LOOP FIXED-DIMENSION PERTURBATIVE EXPANSION.}

\section{A. The six-loop series}

In the fixed-dimension field-theoretical approach, see, e.g., Ref. [4] and references therein, one expands in powers of appropriately defined zero-momentum quartic couplings. In the present case we define renormalized couplings $\bar{u}, \bar{v}$, and $\bar{w}$ from the zero-momentum fourpoint function. They are normalized so that, at tree level, they are related to the bare couplings $u, v$, and $w$ by

$$
u=\frac{16 \pi}{3} R_{M N} \bar{u} m, \quad v=\frac{16 \pi}{3} R_{M} \bar{v} m, \quad w=\frac{16 \pi}{3} \bar{w} m
$$

where $R_{K}=9 /(8+K)$ and $m$ is the renormalized mass defined by 


$$
\Gamma_{a i, b j}^{(2)}(p)=\delta_{a i, b j} Z_{\phi}^{-1}\left[m^{2}+p^{2}+O\left(p^{4}\right)\right]
$$

Here $\Gamma_{a i, b j}^{(2)}(p)$ is the two-point one-particle irreducible correlation function.

By using the numerical results compiled in Ref. [37] and a general symbolic manipulation program, we determined the RG functions to six loops. The resulting series for $N=0$ are

$$
\begin{gathered}
\beta_{u}=m \frac{\partial \bar{u}}{\partial m}=-\bar{u}+\bar{u}^{2}+\frac{2(M+2)}{(M+8)} \bar{u} \bar{v}+\frac{2}{3} \bar{u} \bar{w}-\frac{95}{216} \bar{u}^{3}-\frac{50(M+2)}{27(M+8)} \bar{u}^{2} \bar{v}-\frac{92(M+2)}{27(M+8)^{2}} \bar{u} \bar{v}^{2} \\
-\frac{50}{81} \bar{u}^{2} \bar{w}-\frac{92}{729} \bar{u} \bar{w}^{2}-\frac{184}{81(M+8)} \bar{u} \bar{v} \bar{w}+\bar{u}\left(\sum_{i+j+k \geq 3} b_{i j k}^{(u)} \bar{u}^{i} \bar{v}^{j} \bar{w}^{k}\right), \\
\beta_{v}=m \frac{\partial \bar{v}}{\partial m}=-\bar{v}+\bar{v}^{2}+\frac{3}{2} \bar{u} \bar{v}+\frac{2}{3} \bar{v} \bar{w}-\frac{4(190+41 M)}{27(M+8)^{2}} \bar{v}^{3}-\frac{2(131+25 M)}{27(M+8)} \bar{u}^{2} \\
-\frac{185}{216} \bar{u}^{2} \bar{v}-\frac{400}{81(M+8)} \bar{v}^{2} \bar{w}-\frac{92}{729} \bar{v} \bar{w}^{2}-\frac{77}{81} \bar{u} \bar{v} \bar{w}+\bar{v}\left(\sum_{i+j+k \geq 3} b_{i j k}^{(v)} \bar{u}^{i} \bar{v}^{j} \bar{w}^{k}\right), \\
\beta_{w}=m \frac{\partial \bar{v}}{\partial m}=-\bar{w}+\bar{w}^{2}+\frac{3}{2} \bar{u} \bar{w}+\frac{12}{(M+8)} \bar{v} \bar{w}-\frac{308}{729} \bar{w}^{3}-\frac{104}{81} \bar{u} \bar{w}^{2}-\frac{832}{81(M+8)} \bar{v} \bar{w}^{2} \\
-\frac{555}{648} \bar{u}^{2} \bar{w}-\frac{4(370+23 M)}{27(M+8)^{2}} \bar{v}^{2} \bar{w}-\frac{(23 M+370)}{27(M+8)} \bar{u} \bar{v} \bar{w}+\bar{w}\left(\sum_{i+j+k \geq 3} b_{i j k}^{(w)} \bar{u}^{i} \bar{v}^{j} \bar{w}^{k}\right) .
\end{gathered}
$$

The coefficients $b_{i j k}^{(u)}, b_{i j k}^{(v)}, b_{i j k}^{(w)}$, with $3 \leq i+j+k \leq 6$, are reported in the Tables III, IV, and $\mathrm{V}$, respectively. We have also computed the $\mathrm{RG}$ functions associated with the critical exponents to six loops. We do not report them either since they will not be used in our analysis (they are available on request).

\section{B. Resummation of the series}

The perturbative series for the $M$-vector model and for the cubic model are Borel summable and thus accurate results can be obtained by resummation methods that exploit Borel summability and the knowledge of the large-order behavior $[38,7]$. On the other hand, perturbative series for randomly dilute models cannot be resummed naively. In the present case, an extension of the results of Refs. [39,40] indicates that the perturbative series at fixed $u / v$ and $u / w$ are not Borel summable. For the zero-dimensional random Ising model, Ref. [41] showed that one could still compute correctly the free energy by means of a more elaborate resummation method. Although no proof exists that this procedure works in higher dimensions, this method was recently applied to resum six-loop expansions for the random Ising model in three dimensions [31], obtaining reasonably accurate results.

Here, we apply a similar method to resum the perturbative series for the randomly dilute cubic model for generic values of $u, v, w$. If $f(u, v, w)$ has a perturbative expansion of the form 


$$
\begin{aligned}
& f(u, v, w)=\sum_{n=0} c_{n}(v, w) u^{n}, \\
& c_{n}(v, w)=\sum_{k, l=0} c_{n k l} v^{k} w^{l},
\end{aligned}
$$

following Ref. [31], we first resum the coefficients $c_{n}(v, w)$ and then, using the computed coefficients, we resum the series in $u$. The first resummation can be performed by using either the Padé-Borel method or the conformal-mapping method-indeed, the large-order behavior of the coefficients $c_{n}(v, w)$ is exactly the one of the $M$-component cubic model [7]. In the Padé-Borel method, for each $0 \leq n \leq p$ (where $p$ is maximum order considered), we consider the Padé approximants $\left[\left(p-n-r_{n}\right) / r_{n}\right]$ of the Borel-Leroy transformed series, which depend on an additional parameter $b_{n}$. The second method uses the large-order behavior of the series and a conformal mapping, see Ref. [31]. Once an estimate of the coefficients $c_{n}(v, w)$ is obtained, the second resummation is performed by using the PadéBorel method. We consider Padé approximants $\left[\left(q-r_{u}\right) / r_{u}\right]$ of the Borel-Leroy transformed series, with $q \leq p-1$. Therefore, in the double Padé-Borel method, which we will mostly use, the parameters introduced by the analysis are $p,\left\{b_{n}\right\},\left\{r_{n}\right\}$ for the first resummation and $q, b_{u}, r_{u}$ for the second one. It is impossible to vary all the $b_{n}$ and $r_{n}$ independently, since there are too many combinations. Therefore, following Ref. [31], we took them equal for all $n$.

In the special cases when one of the coupling vanishes, one may use the methods already applied in the literature for the study of the corresponding model. For example, the series for $u=0$ correspond to those of the Hamiltonian (1.3); they are Borel summable, so that one may use the standard technique based on the knowledge of the large-order behavior and a conformal mapping [7]. The series for $v=0$ correspond to those of the random Ising Hamiltonian (1.3) with $M \rightarrow 0$; thus, one may use the analysis methods outlined in Ref. [31].

\section{Results}

\section{Stability of the fixed points of the pure systems}

First, we check the stability of the stable fixed point of the pure theory $(\bar{u}=0)$ with respect to the perturbation induced by random dilution.

The coordinates of the stable $\mathrm{O}(2)$-symmetric fixed point of the two-component theory are $[27,42] \bar{u}=0, \bar{v}_{X Y}=1.402(4)$, and $\bar{w}=0$. The stability with respect to random dilution is controlled by

$$
\omega_{u}=\left.\frac{\partial \beta_{u}}{\partial \bar{u}}\right|_{\left(0, \bar{v}_{X Y}, 0\right)},
$$

which, according to the nonpertubative argument reported in Sec. II, should be given by

$$
\omega_{u}=-\frac{\alpha_{X Y}}{\nu_{X Y}},
$$

where $\alpha_{X Y}$ and $\nu_{X Y}$ are the critical exponents of the $X Y$ model. The analysis of the series (exploiting the known large-order behavior of the series and using the conformalmapping method) gives $\omega_{u}=0.007(8)$. Therefore, the stability of the $X Y$ fixed point 
TABLE II. Estimates of the subleading exponent $\omega_{u}$ at the $M$-component cubic fixed point for several values of $M$. The last column reports the theoretical prediction $-\alpha_{c} / \nu_{c}$ obtained from the results of Ref. [7]. For $M=3$ we also report the number obtained from the estimates (2.2).

\begin{tabular}{cccc}
\hline \hline$M$ & {$\left[\bar{v}_{c}, \bar{w}_{c}\right]$} & $\omega_{u}$ & $-\alpha_{c} / \nu_{c}$ \\
\hline \hline 3 & {$[1.321(18), 0.096(20)]$} & $0.157(28)$ & $0.167(24), 0.187(3)$ \\
4 & {$[0.881(14), 0.639(14)]$} & $0.203(27)$ & $0.199(30)$ \\
8 & {$[0.440(12), 1.136(10)]$} & $0.199(21)$ & $0.191(24)$ \\
$\infty$ & {$[0.174(6), 1.417(6)]$} & $0.179(16)$ & $0.175(30)$ \\
\hline \hline
\end{tabular}

is substantially confirmed, although the apparent error of the analysis does not completely exclude the opposite sign for $\omega_{u}$. The estimate of $\omega_{u}$ is substantially consistent with Eq. (4.9). Indeed, $\alpha_{X Y} / \nu_{X Y}=-0.0217(12)$ (Ref. [27]), obtained from the analysis of high-temperature series, and [42] $\alpha_{X Y} / \nu_{X Y}=-0.016(7)$, obtained by a more similar technique, i.e., the analysis of the fixed-dimension expansion of the $\mathrm{O}(2)$-symmetric model. We mention that perturbative studies based on shorter series $[43,44]$ give apparently contradictory results, favoring a negative value of $\omega_{u}$.

We now consider the case $M \geq 3$. The relevant fixed point is now the cubic one with coordinates $\left(0, \bar{v}_{c}, \bar{w}_{c}\right)$. The stability against the $\bar{u}$ perturbation is determined by the sign of

$$
\omega_{u}=\left.\frac{\partial \beta_{u}}{\partial \bar{u}}\right|_{\left(0, \bar{v}_{c}, \bar{w}_{c}\right)} .
$$

Again, one expects $\omega_{u}=-\alpha_{c} / \nu_{c}$, where $\alpha_{c}$ and $\nu_{c}$ are the critical exponents associated with the cubic fixed point. Since the large-order behavior of the expansion of $\omega_{u}$ is that of the series of the cubic-symmetric pure model, one can use the standard conformal-mapping resummation [7]. Estimates of $\omega_{u}$ for several values of $M$ are reported in Table II (we use the estimates of the cubic fixed points reported in Ref. [7]). For comparison, we also report the ratio $-\alpha_{c} / \nu_{c}$ as obtained from the results of Ref. [7]. Other estimates can be obtained using the results of Refs. [6,45]. For $M=3$, we also quote $-\alpha_{c} / \nu_{c} \approx 0.142[45],-\alpha_{c} / \nu_{c} \approx 0.163$ [6].

In conclusion, the analysis of the six-loop perturbative series shows that the stable fixed point of the system without disorder is stable with respect to the addition of random impurities, in agreement with the Harris criterion.

\section{Fixed points induced by the disorder, i.e. for $u<0$.}

We now study the stability of the RIM fixed point located in the plane $v=0$. Its coordinates are [31] $\bar{u}_{\mathrm{RIM}}=-0.631(16), \bar{v}=0, \bar{w}_{\mathrm{RIM}}=2.195(20)$. This fixed point is stable in the plane $v=0$. To check its stability with respect to the $v$-perturbation we need to compute only

$$
\omega_{v}=\left.\frac{\partial \beta_{v}}{\partial \bar{v}}\right|_{\left(\bar{u}_{\left.\mathrm{RIM}, 0, \bar{w}_{\mathrm{RIM}}\right)},\right.},
$$


since the derivatives of $\beta_{v}$ with respect to $\bar{u}$ and $\bar{w}$ vanish at $\bar{v}=0$. Note that the series for $\omega_{v}$ is $M$ independent. We apply the double Padé-Borel method to resum the series. We find that nondefective Padé approximants are obtained only for $r_{w}=1$. Setting $q=p-1$, the results depend on four free parameters: $p, r_{u}, b_{u}$, and $b_{w}$. In principle, we should look for the values of $b_{u}$ and $b_{w}$ that make the estimates independent of the order $p$ of the series, but in the present case the results are weakly depending upon these two parameters. So, we consider all values $0 \leq b_{u}, b_{w} \leq 10$. With this choice, all approximants with $r_{u}=1$ and $r_{u}>2$ are defective and therefore, we use $p=4,5$, and 6 and $r_{u}=0,2$. For $r_{u}=0$ (direct summation) we obtain $\omega_{v}=-0.047(8)\{20\}$ for $p=4, \omega_{v}=0.008(2)\{20\}$ for $p=5$, and $\omega_{v}=-0.049(2)\{20\}$ for $p=6$, where the number between parentheses is related to the spread of the results of the approximants considered, while the one between braces is related to the uncertainty on the location of the fixed point. For $r_{u}=2$, all approximants with $p=4$ are defective. For $p=5$ we obtain $\omega_{v}=-0.012(14)\{20\}$ and for $p=6 \omega_{v}=-0.080(5)\{20\}$. The quite large discrepancy between these estimates clearly indicates that the analysis is not very robust. We give as conservative final estimate $\omega_{v}=-0.04(5)$, that includes all previous results [46]. This value suggests that the RIM fixed point is unstable, although it does not allow us to exclude the opposite case.

Finally, we search for the presence of new fixed points in the physical region $\bar{u}<0$. Our analysis does not provide evidence for new fixed points in the physical region $\bar{u}<0$, at least for $\bar{u} \gtrsim-1$, the region in which sufficiently stable results are obtained. In the case $M=2$, we only find the fixed point predicted by the symmetry (2.3). Indeed, a fixed point equivalent to the RIM is expected at $\bar{u}^{*}=\bar{u}_{\mathrm{RIM}}^{*}=-0.631(16), \bar{v}^{*}=\bar{v}_{\mathrm{RIM}}^{*}+\frac{5}{3} \bar{w}_{\mathrm{RIM}}^{*}=3.658(33)$, $\bar{w}^{*}=-\bar{w}_{\mathrm{RIM}}^{*}=-2.195(20)$. Our resummation of the six-loop series gives consistent results for the location of the fixed point, i.e. $\bar{u}^{*}=-0.70(5), \bar{v}^{*}=3.7(3), \bar{w}^{*}=-2.2(2)$.

\section{ACKNOWLEDGMENT}

We thank Yu. Holovatch for useful correspondence.

\section{APPENDIX A: DIFFERENCES BETWEEN CUBIC AND HEISENBERG CRITICAL EXPONENTS}

In the three-component case the cubic critical exponents differ very little from those of the Heisenberg universality class. The available estimates of the critical exponents are indistinguishable within their uncertainty. Field-theoretical six-loop calculations for the cubic model [7] give $\nu_{c}=0.706(6), \eta_{c}=0.0333(26)$ and $\gamma_{c}=1.390(12)$, while the analysis of Ref. [42] of six- and seven-loop series for the $\mathrm{O}(3)$-symmetric Heisenberg model provide the estimates $\nu_{H}=0.7073(35), \eta_{H}=0.0355(25), \gamma_{H}=1.3895(50)$. We also mention the more accurate estimates $\nu_{H}=0.7112(5), \eta_{H}=0.0375(5), \gamma_{H}=1.3960(9)$, obtained by lattice techniques [26]. By comparing these estimates we can only put a bound on the differences of the cubic and Heisenberg exponents; for example, the estimates of $\nu$ differ at most by $1 \%$.

Much better estimates of such differences can be obtained by a more careful analysis of the six-loop fixed-dimension series of the cubic model (1.3) computed in Ref. [7]. We recall that the expansion is performed in powers of the zero-momentum quartic couplings $\bar{v}$ and 
$\bar{w}$ associated respectively with the couplings $v, w$ of the cubic Hamiltonian (1.3). The series for the cubic model can be obtained by setting $\bar{u}=0$ in the series reported in Sec. IV. In the plane $\bar{v}, \bar{w}$ the coordinates of the $\mathrm{O}(3)$-symmetric and cubic fixed points are respectively $[42,26] X_{H}=[1.390(4), 0]$ and $[7] X_{c}=[1.321(18), 0.096(20)]$. In order to obtain a more precise determination of the exponents, one may proceed as follows. Noting the closeness of the fixed points, one may estimate the difference of the corresponding critical esponents $\eta$ and $\nu$ by expanding around the Heisenberg fixed point. Therefore, the first-order approximation is

$$
\begin{aligned}
& \Delta \eta \equiv \eta_{c}-\eta_{H} \approx \Delta \eta^{(1)}=\left.\frac{\partial \eta_{\phi}}{\partial \bar{v}}\right|_{X_{H}}\left(\bar{v}_{c}-\bar{v}_{H}\right)+\left.\frac{\partial \eta_{\phi}}{\partial \bar{w}}\right|_{X_{H}} \bar{w}_{c} \\
& \Delta \nu \equiv \nu_{c}-\nu_{H} \approx \Delta \nu^{(1)}=\left.\frac{\partial \nu}{\partial \bar{v}}\right|_{X_{H}}\left(\bar{v}_{c}-\bar{v}_{H}\right)+\left.\frac{\partial \nu}{\partial \bar{w}}\right|_{X_{H}} \bar{w}_{c} .
\end{aligned}
$$

The RG functions $\eta_{\phi}(\bar{v}, \bar{w}), \eta_{t}(\bar{v}, \bar{w})$, and $\nu(\bar{v}, \bar{w})=\left(2-\eta_{\phi}+\eta_{t}\right)^{-1}$ have been introduced in Ref. [7]. The expressions (A1) can be simplified using the relation [47]

$$
\left.\frac{\partial \eta_{\phi, t}}{\partial \bar{w}}\right|_{(\bar{v}, 0)}=\left.\frac{M+8}{3(M+2)} \frac{\partial \eta_{\phi, t}}{\partial \bar{v}}\right|_{(\bar{v}, 0)},
$$

which is verified by our six-loop series and is probably exact. Therefore, we write

$$
\begin{aligned}
& \Delta \eta^{(1)}=\left.\frac{\partial \eta_{\phi}}{\partial \bar{v}}\right|_{X_{H}}\left(\bar{v}_{c}-\bar{v}_{H}+\frac{11}{15} \bar{w}_{c}\right), \\
& \Delta \nu^{(1)}=\left.\frac{\partial \nu}{\partial \bar{v}}\right|_{X_{H}}\left(\bar{v}_{c}-\bar{v}_{H}+\frac{11}{15} \bar{w}_{c}\right) .
\end{aligned}
$$

In order to estimate the right-hand side of the above equations, one needs to consider all the elements of the covariance matrix associated with the cubic fixed point $X_{c}$, i.e. $C_{\bar{u} \bar{u}}=0.000345, C_{\bar{v} \bar{v}}=0.000380$, and $C_{\bar{u} \bar{v}}=-0.000361$, because the estimates of the coordinates $\bar{v}_{c}, \bar{w}_{c}$ are strongly correlated. Using the covariance matrix one obtains the quite precise estimate $\bar{v}_{c}+\frac{11}{15} \bar{w}_{c}=1.391(4)$, which is very close to $\bar{v}_{H}=1.390(4)$, thus leading to a large cancellation in the right-hand side of Eqs. (A3). In alternative, one may consider the approximate relation

$$
\left.\frac{\partial \beta_{v}}{\partial \bar{v}}\right|_{X_{H}}\left(\bar{v}_{c}-\bar{v}_{H}\right)+\left.\frac{\partial \beta_{v}}{\partial \bar{w}}\right|_{X_{H}} \bar{w}_{c} \approx 0,
$$

which is obtained by expanding the equation $\beta_{v}\left(\bar{v}_{c}, \bar{w}_{c}\right)=0$. Within this approximation, one obtains $\bar{v}_{c}-\bar{v}_{H}=-0.743(10) \bar{v}_{c}$, and therefore $\bar{v}_{c}-\bar{v}_{H}+\frac{11}{15} \bar{w}_{c}=-0.0009(8)$. Moreover, the analysis of the series provides the results $\partial \eta_{\phi} /\left.\partial \bar{v}\right|_{X_{H}}=0.06(1)$ and $\partial \nu /\left.\partial \bar{v}\right|_{X_{H}}=0.21(1)$. Inserting in Eqs. (A3), we finally obtain $\Delta \eta^{(1)}=-0.00005(5)$ and $\Delta \nu^{(1)}=-0.0002(2)$. Next, we determined the second-order contributions to Eq. (A1). It can be estimated by evaluating the second derivatives of $\eta_{\phi}, \eta_{t}$, and $\beta_{u}$ at $X_{H}$. We obtained $\Delta \eta^{(2)} \approx-0.00002$ and $\Delta \nu^{(2)} \approx-0.0001$. We also checked that the third-order contributions are very small and negligible. Summing up, we obtain the estimates (2.1). 
TABLE III. The coefficients $b_{i j k}^{(u)}$, cf. Eq. (4.3).

\begin{tabular}{|c|c|}
\hline$i, j, k$ & $R_{M}^{-j} b_{i j k}^{(u)}$ \\
\hline $0,0,3$ & 0.090449 r \\
\hline $0,1,2$ & $\begin{array}{l}0.266228+0.00511843 M \\
0.23096+0.0403865 M\end{array}$ \\
\hline $\begin{array}{l}0,2,1 \\
0,3,0\end{array}$ & $\begin{array}{l}0.23096+0.0403865 M \\
0.0513245+0.034637 M+0.00448739 M^{2}\end{array}$ \\
\hline $1,0,2$ & 0.467389 \\
\hline $1,1,1$ & $0.844729+0.0900481 M$ \\
\hline $1,2,0$ & $0.281576+0.170804 M+0.015008 M^{2}$ \\
\hline $2,0,1$ & 0.857364 r perzee \\
\hline $\begin{array}{l}2,1,0 \\
3,0,0\end{array}$ & $\begin{array}{l}0.571576+0.285788 M \\
0.389923\end{array}$ \\
\hline $0,0,4$ & -0.0754467 \\
\hline $0,1,3$ & $-0.298245-0.00354189 M$ \\
\hline $0,2,2$ & $-0.434652-0.0180279 M$ \\
\hline $0,3,1$ & $-0.24561-0.0570548 M+0.000878001 M^{2}$ \\
\hline $0,4,0$ & $-0.040935-0.0299766 M-0.00460823 M^{2}+0.0000731668 M^{3}$ \\
\hline $1,0,3$ & -0.476234 \\
\hline $1,1,2$ & $-1.38978-0.0389194 M$ \\
\hline $1,2,1$ & $-1.18263-0.250819 M+0.00474531 M^{2}$ \\
\hline $1,3,0$ & $-0.262806-0.187141 M-0.0268143 M^{2}+0.000527256 M^{3}$ \\
\hline $\begin{array}{l}2,0,2 \\
2,1,1\end{array}$ & -1.2213 \\
\hline $2,1,1$ & \\
\hline $2,2,0$ & $-0.699479-0.464461 M-0.0573609 M^{2}$ \\
\hline $\begin{array}{l}3,0,1 \\
3,10\end{array}$ & $\begin{array}{l}-1.34386 \\
-0.895907-0.447954 M\end{array}$ \\
\hline $\begin{array}{l}3,1,0 \\
4,0,0\end{array}$ & $\begin{array}{l}-0.895907-0.447954 M \\
-0.447316\end{array}$ \\
\hline$\frac{4,0,0}{0,0,5}$ & $\frac{-0.447316}{0.0874933}$ \\
\hline & $\begin{array}{l}0.0874933 \\
0.434773+0.0026939 M\end{array}$ \\
\hline $0,2,3$ & $\begin{array}{l}0.434773+0.0026939 M \\
0.856086+0.0188466 M\end{array}$ \\
\hline $0,3,2$ & $\begin{array}{l}0.856086+0.0188466 M \\
0.805412+0.0689363 M+0.000584428 M^{2}\end{array}$ \\
\hline $0,4,1$ & $\begin{array}{l}0.805412+0.0689363 M+0.000584428 M^{2} \\
0.334915+0.0984617 M+0.00404184 M^{2}+0.0000476511 M^{3}\end{array}$ \\
\hline $0,5,0$ & $0.0446554+0.0354559 M+0.00710302 M^{2}+0.000275809 M^{3}+3.17674 \times 10^{-6} M^{4}$ \\
\hline $1,0,4$ & 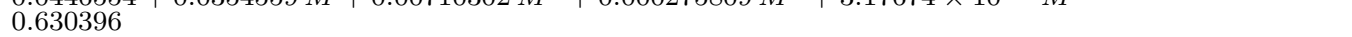 \\
\hline $1,1,3$ & $2.48473+0.0368538 M$ \\
\hline $1,2,2$ & $3.52129+0.258766 M+0.00231537 M^{2}$ \\
\hline $1,3,1$ & $1.95691+0.546998 M+0.0172536 M^{2}+0.00042021 M^{3}$ \\
\hline $1,4,0$ & $0.326152+0.254242 M+0.0484588 M^{2}+0.00150784 M^{3}+0.0000350175 M^{4}$ \\
\hline $2,0,3$ & $1.93839 \quad-10$ \\
\hline $2,1,2$ & $5.51853+0.296649 M$ \\
\hline $2,2,1$ & $4.61152+1.18578 M+0.0178836 M^{2}$ \\
\hline $2,3,0$ & $1.02478+0.775897 M+0.135727 M^{2}+0.00198707 M^{3}$ \\
\hline $3,0,2$ & 3.14287 \\
\hline $3,1,1$ & $5.26405+1.02168 M$ \\
\hline $3,2,0$ & $1.75468+1.2179 M+0.17028 M^{2}$ \\
\hline $4,0,1$ & 2.43619 \\
\hline $4,1,0$ & $1.62413+0.812063 M$ \\
\hline $5,0,0$ & 0.633855 \\
\hline $0,0,6$ & -0.117951 \\
\hline $0,1,5$ & $-0.703992-0.00371266 M$ \\
\hline $0,2,4$ & $-1.74808-0.0211855 M$ \\
\hline $0,3,3$ & $-2.2733-0.0857507 M+0.0000391428 M^{2}$ \\
\hline $0,4,2$ & $-1.57598-0.192268 M-0.00103889 M^{2}+0.0000241287 M^{3}$ \\
\hline $0,5,1$ & $-0.517589-0.178763 M-0.0115596 M^{2}+0.000202814 M^{3}+3.35858 \times 10^{-6} M^{4}$ \\
\hline $0,6,0$ & $-0.0575099-0.0486174 M-0.0112156 M^{2}-0.000619664 M^{3}+0.0000116406 M^{4}+1.86588 \times 10^{-7} M^{5}$ \\
\hline $1,0,5$ & -0.970081 \\
\hline $1,1,4$ & $-4.81696-0.0334495 M$ \\
\hline $1,2,3$ & $-9.40257-0.29895 M+0.00070522 M^{2}$ \\
\hline $1,3,2$ & $-8.70366-0.994183 M-0.00319474 M^{2}+0.00022649 M^{3}$ \\
\hline $1,4,1$ & $-3.57656-1.20315 M-0.0721365 M^{2}+0.00139263 M^{3}+0.0000411225 M^{4}$ \\
\hline $1,5,0$ & $-0.476875-0.398857 M-0.0898279 M^{2}-0.00462342 M^{3}+0.000098325 M^{4}+2.7415 \times 10^{-6} M^{5}$ \\
\hline $2,0,4$ & 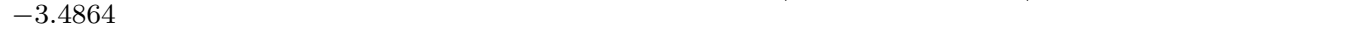 \\
\hline $2,1,3$ & $-13.6166-0.329002 M$ \\
\hline $2,2,2$ & $-18.9348-1.97645 M-0.00712793 M^{2}$ \\
\hline $2,3,1$ & $-10.382-3.38062 M-0.185104 M^{2}+0.00215554 M^{3}$ \\
\hline $2,4,0$ & $-1.73034-1.4286 M-0.312569 M^{2}-0.0150661 M^{3}+0.000179628 M^{4}$ \\
\hline $3,0,3$ & 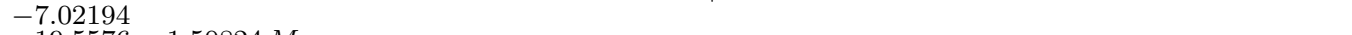 \\
\hline $3,1,2$ & $-19.5576-1.50824 M$ \\
\hline $3,2,1$ & $-16.0889-4.7868 M-0.190108 M^{2}$ \\
\hline $3,3,0$ & $-3.57531-2.85139 M-0.574113 M^{2}-0.0211231 M^{3}$ \\
\hline $4,0,2$ & -8.19093 \\
\hline $4,1,1$ & $-13.4675-2.91439 M$ \\
\hline $4,2,0$ & $-4.48915-3.21604 M-0.485732 M^{2}$ \\
\hline $5,0,1$ & -4.86262 \\
\hline $\begin{array}{l}5,1,0 \\
6,0,0\end{array}$ & $\begin{array}{l}-3.24175-1.62087 M \\
-1.03493\end{array}$ \\
\hline
\end{tabular}


TABLE IV. The coefficients $b_{i j k}^{(v)}$, cf. Eq. (4.4).

\begin{tabular}{|c|c|}
\hline$i, j, k$ & $R_{M}^{-j-1} b_{i j k}^{(v)}$ \\
\hline$\overline{\overline{0,0,3}}$ & $0.0803991+0.0100499 M$ \\
\hline $0,1,2$ & $0.369295+0.0484367 M+0.000284357 M^{2}$ \\
\hline $0,2,1$ & $0.602154+0.0995834 M+0.00303927 M^{2}$ \\
\hline $0,3,0$ & $0.243427+0.0974186 M+0.0100186 M^{2}+0.0002056 M^{3}$ \\
\hline $1,0,2$ & $0.564685+0.0705856 M$ \\
\hline $1,1,1$ & $1.80131+0.266837 M+0.00520921 M^{2}$ \\
\hline $1,2,0$ & $1.0441+0.397331 M+0.0362666 M^{2}+0.000364277 M^{3}$ \\
\hline $2,0,1$ & $1.44157+0.180197 M$ \\
\hline $2,1,0$ & $1.56695+0.524919 M+0.0411313 M^{2}$ \\
\hline$\frac{3,0,0}{0,0,4}$ & $\frac{0.814816+0.101852 M}{-0.0670637-0.00838297 M}$ \\
\hline $0,1,3$ & $-0.376283-0.0486096 M-0.000196771 M^{2}$ \\
\hline $0,2,2$ & $-0.857759-0.118296 M-0.00138446 M^{2}$ \\
\hline $0,3,1$ & $-0.838966-0.178873 M-0.00870044 M^{2}+0.0000687335 M^{3}$ \\
\hline $0,4,0$ & $-0.248229-0.112659 M-0.0150567 M^{2}-0.000585531 M^{3}+2.63588 \times 10^{-6} M^{4}$ \\
\hline $1,0,3$ & $-0.548393-0.0685491 M$ \\
\hline $1,1,2$ & $-2.46028-0.326888 M-0.00241906 M^{2}$ \\
\hline $1,2,1$ & $-3.52974-0.717174 M-0.0328982 M^{2}+0.000199551 M^{3}$ \\
\hline $1,3,0$ & $-1.35535-0.598611 M-0.0763386 M^{2}-0.00279075 M^{3}+5.68096 \times 10^{-6} M^{4}$ \\
\hline $2,0,2$ & $-1.86702-0.233378 \mathrm{M}$ \\
\hline $2,1,1$ & $-5.18827-0.965409 M-0.0396094 M^{2}$ \\
\hline $2,2,0$ & $-2.87884-1.21126 M-0.142369 M^{2}-0.0044929 M^{3}$ \\
\hline $3,0,1$ & $-2.67974-0.334967 M$ \\
\hline $3,1,0$ & $-2.83487-0.985509 M-0.0788938 M^{2}$ \\
\hline $4,0,0$ & $-1.09216-0.136521 \mathrm{M}$ \\
\hline $0,0,5$ & $0.0777718+0.00972148 M$ \\
\hline $0,1,4$ & $0.498091+0.0634586 M+0.000149661 M^{2}$ \\
\hline $0,2,3$ & $1.36139+0.179665 M+0.00118632 M^{2}$ \\
\hline $0,3,2$ & $1.96208+0.303336 M+0.00757647 M^{2}+0.0000396103 M^{3}$ \\
\hline $0,4,1$ & $1.35191+0.341883 M+0.0225965 M^{2}+0.000151344 M^{3}+3.53109 \times 10^{-6} M^{4}$ \\
\hline $0,5,0$ & $0.312662+0.156794 M+0.0247779 M^{2}+0.00130674 M^{3}+6.86391 \times 10^{-6} M^{4}+9.64099 \times 10^{-8} M^{5}$ \\
\hline $1,0,4$ & $0.685931+0.0857414 M$ \\
\hline $1,1,3$ & $3.73862+0.480512 M+0.00164801 M^{2}$ \\
\hline $1,2,2$ & $8.02393+1.1954 M+0.0248927 M^{2}+0.000105193 M^{3}$ \\
\hline $1,3,1$ & $7.26959+1.78276 M+0.110756 M^{2}+0.000298431 M^{3}+0.000013906 M^{4}$ \\
\hline $1,4,0$ & $2.06581+1.01732 M+0.155716 M^{2}+0.00765683 M^{3}+8.6785110^{-6} M^{4}+2.53675 \times 10^{-7} M^{5}$ \\
\hline $2,0,3$ & $2.68432+0.33554 M$ \\
\hline $2,1,2$ & $11.3782+1.62939 M+0.0258891 M^{2}$ \\
\hline $2,2,1$ & $15.1532+3.58071 M+0.208316 M^{2}-0.000313018 M^{3}$ \\
\hline $2,3,0$ & $5.60954+2.7006 M+0.397804 M^{2}+0.0180874 M^{3}-0.0000496743 M^{4}$ \\
\hline $3,0,2$ & $5.6561+0.707013 M$ \\
\hline $3,1,1$ & $14.6374+3.06149 M+0.153977 M^{2}$ \\
\hline $3,2,0$ & $7.87876+3.54347 M+0.468803 M^{2}+0.018622 M^{3}$ \\
\hline $4,0,1$ & $5.56346+0.695432 M$ \\
\hline $4,1,0$ & $5.75768+2.06202 M+0.167789 M^{2}$ \\
\hline $5,0,0$ & $1.75644+0.219555 M$ \\
\hline $0,0,6$ & $-0.104845-0.0131056 M$ \\
\hline $0,1,5$ & $-0.766484-0.0974606 M-0.000206259 M^{2}$ \\
\hline $0,2,4$ & $-2.44861-0.314595 M-0.00106485 M^{2}$ \\
\hline $0,3,3$ & $-4.38376-0.608037 M-0.00748317 M^{2}+3.14201 \times 10^{-6} M^{3}$ \\
\hline $0,4,2$ & $-4.54538-0.806121 M-0.0303046 M^{2}-0.0000528742 M^{3}+2.15704 \times 10^{-6} M^{4}$ \\
\hline $0,5,1$ & $-2.39859-0.70043 M-0.0596949 M^{2}-0.00115086 M^{3}+8.26434 \times 10^{-6} M^{4}+2.2795 \times 10^{-7} M^{5}$ \\
\hline $0,6,0$ & $-0.453777-0.247702 M-0.0450631 M^{2}-0.00309346 M^{3}-0.000053787 M^{4}+$ \\
\hline $\begin{array}{l}1,0,0 \\
1,1,4\end{array}$ & $\begin{array}{l}-1.0206-0.127575 M \\
-6.51597-0.822062 M-0.000945689 M^{2}\end{array}$ \\
\hline $1,2,3$ & $-17.4536-2.36625 M-0.0229946 M^{2}+9.24619 \times 10^{-6} M^{3}$ \\
\hline $1,3,2$ & $-23.9918-4.14517 M-0.145391 M^{2}-0.000191685 M^{3}+9.11727 \times 10^{-6} M^{4}$ \\
\hline $1,4,1$ & $-15.6729-4.48444 M-0.369036 M^{2}-0.00652617 M^{3}+0.000027074 M^{4}+1.11884 \times 10^{-6} M^{5}$ \\
\hline $1,5,0$ & $-3.51598-1.89485 M-0.337579 M^{2}-0.0223394 M^{3}-0.000357755 M^{4}+$ \\
\hline & $+4.31553 \times 10^{-7} M^{5}+1.52437 \times 10^{-8} M^{6}$ \\
\hline $2,0,4$ & $-4.4801-0.560012 M$ \\
\hline $2,1,3$ & $-23.8866-3.18735 M-0.0251901 M^{2}$ \\
\hline $2,2,2$ & $-48.8238-8.24755 M-0.271114 M^{2}-0.000380338 M^{3}$ \\
\hline $2,3,1$ & $-41.9591-11.7782 M-0.936933 M^{2}-0.0151248 M^{3}-0.0000114803 M^{4}$ \\
\hline $2,4,0$ & $-11.5837-6.16458 M-1.07549 M^{2}-0.0685196 M^{3}-0.000997932 M^{4}-3.16906 \times 10^{-6} M^{5}$ \\
\hline $3,0,3$ & $-11.3503-1.41879 M$ \\
\hline $3,1,2$ & $-45.7836-7.2337 M-0.188844 M^{2}$ \\
\hline $3,2,1$ & $-57.9214-15.4564 M-1.13636 M^{2}-0.0136677 M^{3}$ \\
\hline $3,3,0$ & $-20.8832-10.7783 M-1.79165 M^{2}-0.105048 M^{3}-0.00108945 M^{4}$ \\
\hline $4,0,2$ & $-16.8465-2.10582 M$ \\
\hline $4,1,1$ & $-41.5421-9.44192 M-0.531144 M^{2}$ \\
\hline $4,2,0$ & $-21.8649-10.3417 M-1.48269 M^{2}-0.066452 M^{3}$ \\
\hline $5,0,1$ & $-12.4665-1.55831 M$ \\
\hline $5,1,0$ & $-12.683-4.64788 M-0.382814 M^{2}$ \\
\hline $6,0,0$ & $-3.1978-0.399725 \mathrm{M}$ \\
\hline
\end{tabular}


TABLE V. The coefficients $b_{i j k}^{(w)}$, cf. Eq. (4.5).

\begin{tabular}{|c|c|}
\hline$i, j, k$ & $R_{M}^{-j} b_{i j k}^{(w)}$ \\
\hline$\overline{0,0,3}$ & 0.35107 \\
\hline $0,2,1$ & $\begin{array}{l}1.31383 \\
1.68533+0.00307141 M\end{array}$ \\
\hline $0,3,0$ & $0.643805+0.0574128 M-0.0017162 M^{2}$ \\
\hline $1,0,2$ & 1.47806 \\
\hline $1,1,1$ & $3.79199+0.00691068 M$ \\
\hline $1,2,0$ & $2.17284+0.193768 M-0.00579216 M^{2}$ \\
\hline $2,0,1$ & 2.133 \\
\hline $\begin{array}{l}2,1,0 \\
3,0,0\end{array}$ & $\begin{array}{l}2.44445+0.199774 M \\
0.916668\end{array}$ \\
\hline $0,0,4$ & -0.376527 \\
\hline $0,1,3$ & -1.80719 \\
\hline $0,2,2$ & $-3.34772+0.00754184 M$ \\
\hline $0,3,1$ & $-2.73858-0.0492189 M-0.0000262347 M^{2}$ \\
\hline $0,4,0$ & $-0.767062-0.0890547 M+0.0000407114 M^{2}-0.0000875861 M^{3}$ \\
\hline $1,0,3$ & -2.03309 \\
\hline $1,1,2$ & $-7.53237+0.0169691 M$ \\
\hline $1,2,1$ & $-9.24272-0.166114 M-0.0000885421 M^{2}$ \\
\hline $1,3,0$ & $-3.45178-0.400746 M+0.000183201 M^{2}-0.000394138 M^{3}$ \\
\hline $2,0,2$ & $\begin{array}{l}-4.23696 \\
-10.3981-0.212414 M\end{array}$ \\
\hline $\begin{array}{l}2,1,1 \\
2\end{array}$ & \\
\hline $\begin{array}{l}2,2,0 \\
3,0,1\end{array}$ & $\begin{array}{l}-5.82488-0.690232 M-0.00248026 M^{2} \\
-3.89927\end{array}$ \\
\hline $\begin{array}{l}3,0,1 \\
3,1,0\end{array}$ & \\
\hline $\begin{array}{l}3,1,0 \\
4,0,0\end{array}$ & $\begin{array}{l}-4.50000-0.410101 M T \\
-1.22868\end{array}$ \\
\hline $0,0,5$ & 0.495548 \\
\hline $0,1,4$ & 2.88579 \\
\hline $0,2,3$ & $6.8946-0.0230874 M$ \\
\hline $0,3,2$ & $8.36453+0.00396206 M+0.000213631 M^{2}$ \\
\hline $0,4,1$ & $4.98655+0.175728 M-0.00207184 M^{2}-0.0000193829 M^{3}$ \\
\hline $0,5,0$ & $1.09653+0.157913 M+0.00235846 M^{2}-0.0000614713 M^{3}-5.38712 \times 10^{-6} M^{4}$ \\
\hline $1,0,4$ & 3.24652 (2) \\
\hline $1,1,3$ & $15.5129-0.0519466 M$ \\
\hline $1,2,2$ & $28.2303+0.0133719 M+0.000721005 M^{2}$ \\
\hline $1,3,1$ & $22.4395+0.790776 M-0.00932327 M^{2}-0.0000872231 M^{3}$ \\
\hline $1,4,0$ & $6.16801+0.88826 M+0.0132664 M^{2}-0.000345776 M^{3}-0.0000303026 M^{4}$ \\
\hline $2,0,3$ & 8.72598 \\
\hline $2,1,2$ & $31.7591+0.124994 M$ \\
\hline $2,2,1$ & $37.8666+1.47045 M-0.0146658 M^{2}$ \\
\hline $2,3,0$ & $13.878+2.04994 M+0.0348703 M^{2}-0.000849221 M^{3}$ \\
\hline $3,0,2$ & $\begin{array}{l}11.9097 \\
28.4+0.965884 M\end{array}$ \\
\hline $3,1,1$ & $\begin{array}{l}28.4+0.965884 M \\
15.6128+2.22247 M+0.0408606 M^{2}\end{array}$ \\
\hline $\begin{array}{l}3,2,0 \\
4,0,1\end{array}$ & $\begin{array}{l}15.6128+2.22247 M+0.0408606 M^{2} \\
7.98749\end{array}$ \\
\hline $\begin{array}{l}4,1,0 \\
5,0,0\end{array}$ & $8.78218+0.933899 M$ \\
\hline$\frac{5,0,0}{0,0.6}$ & 1.97599 \\
\hline $\begin{array}{l}0,0,6 \\
0,1,5\end{array}$ & $\begin{array}{l}-0.749689 \\
-5.12987\end{array}$ \\
\hline $0,2,4$ & $-14.928+0.0486813 M$ \\
\hline $0,3,3$ & $-23.5697+0.0957169 M-0.00083904 M^{2}$ \\
\hline $0,4,2$ & $-21.0735-0.166287 M-0.0000148277 M^{2}+4.49885 \times 10^{-6} M^{3}$ \\
\hline $0,5,1$ & $-9.82983-0.53385 M+0.00220333 M^{2}-0.000130668 M^{3}-2.59594 \times 10^{-6} M^{4}$ \\
\hline $0,6,0$ & $-1.77455-0.304043 M-0.00943381 M^{2}+0.0000669939 M^{3}-6.57249 \times 10^{-6} M^{4}-3.75311 \times 10^{-7} M^{5}$ \\
\hline $1,0,5$ & $\mathbf{l}_{\mathbf{l}}$ \\
\hline $1,1,4$ & $-33.588+0.109533 M$ \\
\hline $1,2,3$ & $-79.5478+0.323044 M-0.00283176 M^{2}$ \\
\hline $1,3,2$ & $-94.8309-0.748291 M-0.0000667246 M^{2}+0.0000202448 M^{3}$ \\
\hline $1,4,1$ & $-55.2928-3.0029 M+0.0123937 M^{2}-0.000735009 M^{3}-0.0000146022 M^{4}$ \\
\hline $1,5,0$ & $-11.9782-2.05229 M-0.0636782 M^{2}+0.000452209 M^{3}-0.0000443643 M^{4}-2.53335 \times 10^{-6} M^{5}$ \\
\hline $2,0,4$ & -18.8932 \\
\hline $2,1,3$ & $-89.4913+0.0651677 M$ \\
\hline $2,2,2$ & $-160.027-1.81428 M+0.00813333 M^{2}$ \\
\hline $2,3,1$ & $-124.409-7.20262 M+0.023168 M^{2}-0.00136807 M^{3}$ \\
\hline $2,4,0$ & $-33.6888-5.89631 M-0.193047 M^{2}+0.00140166 M^{3}-0.000108167 M^{4}$ \\
\hline $3,0,3$ & $-33.5592,-1$, \\
\hline $3,1,2$ & $-120.02-1.44746 \mathrm{M}$ \\
\hline $3,2,1$ & $-139.96-8.18899 M-0.000367593 M^{2}$ \\
\hline $3,3,0$ & $-50.5332-8.87281 M-0.304904 M^{2}+0.00178302 M^{3}$ \\
\hline $4,0,2$ & $-33.7557 \quad 25874 \pi$ \\
\hline $4,1,1$ & $-78.7274-3.5874 M$ \\
\hline $4,2,0$ & $-42.6374-6.91385 M-0.200772 M^{2}$ \\
\hline $5,0,1$ & -17.7137 \\
\hline $5,1,0$ & $-19.1868-2.21571 M$ \\
\hline $6,0,0$ & -3.59753 \\
\hline
\end{tabular}




\section{REFERENCES}

[1] G. Grinstein and A. Luther, Phys. Rev. B 13, 1329 (1976).

[2] A. Aharony, in Phase Transitions and Critical Phenomena, edited by C. Domb and M. S. Green (Academic Press, New York, 1976), Vol. 6, p. 357.

[3] R. B. Stinchcombe, in Phase Transitions and Critical Phenomena, edited by C. Domb and J. Lebowitz (Academic Press, New York, 1983), Vol. 7, p. 152.

[4] A. Pelissetto and E. Vicari, cond-mat/0012164, Phys. Rep. in press.

[5] R. Folk, Yu. Holovatch, and T. Yavors'kii, cond-mat/0106468.

[6] R. Folk, Yu. Holovatch, and T. Yavors'kii, Phys. Rev. B 62, 12195 (2000).

[7] J. M. Carmona, A. Pelissetto, and E. Vicari, Phys. Rev. B 61, 15136 (2000).

[8] K. B. Varnashev, J. Phys. A 33, 3121 (2000); Phys. Rev. B 61, 14660 (2000).

[9] B. N. Shalaev, S. A. Antonenko, and A. I. Sokolov, Phys. Lett. A 230, 105 (1997).

[10] H. Kleinert and V. Schulte-Frohlinde, Phys. Lett. B 342, 284 (1995).

H. Kleinert, S. Thoms, and V. Schulte-Frohlinde, Phys. Rev. B 56, 14428 (1997).

[11] A. B. Harris, J. Phys. C 7, 1671 (1974).

[12] D. Mukamel and G. Grinstein, Phys. Rev. B 25, 381 (1982).

[13] A. Aharony, Phys. Rev. B 12, 1038 (1975).

[14] We mention that the Hamiltonian (1.6) with $M=2$ and $N \geq 2$ is physically interesting because it describes the critical properties in some structural and antiferromagnetic phase transitions, see, e.g., Refs. [48,2]. For recent studies, see, e.g., Refs. [4,43,44] and references therein.

[15] This derivation assumes that the replica symmetry is not broken in the limit $N \rightarrow 0$. This issue is discussed, e.g., in V. S. Dotsenko, J. Phys. A 32, 2949 (1999), V. V. Prudnikov, P.V. Prudnikov, and A. A. Fedorenko, Phys. Rev. B 63, 184201 (2001), and references therein.

[16] Y. Imry and M. Wortis, Phys. Rev. B 19, 3580 (1979).

[17] M. Aizenman and J. Wehr, Phys. Rev. Lett. 62, 2503 (1989).

[18] K. Hui and A. Nihat Berker, Phys. Rev. Lett. 62, 2507 (1989).

[19] J. Cardy, J. Phys. A 29, 1897 (1996).

[20] J. R. Banavar, G. S. Grest, and D. Jasnow, Phys. Rev. Lett. 45, 1424 (1980); Phys. Rev. B 25, 4639 (1982).

[21] M. Itakura, Phys. Rev. B 60, 6558 (1999).

[22] R. K. Heilmann, J. S. Wang, and R. H. Swendsen, Phys. Rev. B 53, 2210 (1996).

M. Kolesik and M. Suzuki, Physica A 216, 469 (1995); J. Phys. A 28, 6543 (1995).

A. P. Gottlob and M. Hasenbusch, Physica A 216, 469 (1994); J. Stat. Phys. 77, 919 (1994).

J. S. Wang, R. H. Swendsen, and R. Kotecký, Phys. Rev. Lett. 63, 109 (1989); Phys. Rev. B 42, 2465 (1990).

[23] S. N. Kaul, J. Magn. Magn. Mater. 53 (1985) 5.

[24] P. D. Babu and S. N. Kaul, J. Phys.: Condens. Matter 9, 7189 (1997).

[25] J. Sak, Phys. Rev. B 10, 3957 (1974).

[26] M. Campostrini, M. Hasenbusch, A. Pelissetto, P. Rossi, and E. Vicari, Phys. Rev. B 65, $144520(2002)$. 
[27] M. Campostrini, M. Hasenbusch, A. Pelissetto, P. Rossi, and E. Vicari, Phys. Rev. B 63, 214503 (2001).

[28] H. G. Ballesteros, L. A. Fernández, V. Martín-Mayor, A. Muñoz Sudupe, G. Parisi, and J. J. Ruiz-Lorenzo, Phys. Rev. B 58, 2740 (1998).

[29] R. Folk, Yu. Holovatch, and T. Yavors'kii, Phys. Rev. B 61, 15114 (2000).

[30] D. V. Pakhnin and A. I. Sokolov, Phys. Rev. B 61, 15130 (2000).

[31] A. Pelissetto and E. Vicari, Phys. Rev. B 62, 6393 (2000).

[32] M. Tissier, D. Mouhanna, J. Vidal, and B. Delamotte, Phys. Rev. B 65, 140402(R) (2002).

[33] C. Jayaprakash and H. J. Katz, Phys. Rev. B 16, 3987 (1977).

[34] D. E. Khmel'nitskii, Zh. Eksp. Teor. Fiz. 68, 1960 (1975) [Sov. Phys. JETP 41, 981 (1975)].

[35] A. Pelissetto, P. Rossi, and E. Vicari, Phys. Rev. B 63, 140414(R) (2001); P. Calabrese and P. Parruccini, Phys. Rev. B 64, 184408 (2001).

[36] B. I. Halperin, T. C. Lubensky, and S. K. Ma, Phys. Rev. Lett. 32, 292 (1974).

S. Mo, J. Hove, and A. Sudbø, Phys. Rev. B 65, 104510 (2002).

[37] B. G. Nickel, D. I. Meiron, and G. A. Baker, Jr., "Compilation of 2-pt and 4-pt graphs for continuum spin models," Guelph University Report, 1977, unpublished.

[38] J. Zinn-Justin, Quantum Field Theory and Critical Phenomena, third edition (Clarendon Press, Oxford, 1996).

[39] A. J. Bray, T. McCarthy, M. A. Moore, J. D. Reger, and A. P. Young, Phys. Rev. B 36, 2212 (1987).

[40] A. J. McKane, Phys. Rev. B 49, 12003 (1994).

[41] G. Álvarez, V. Martín-Mayor, and J. J. Ruiz-Lorenzo, J. Phys. A 33, 841 (2000).

[42] R. Guida and J. Zinn-Justin, J. Phys. A 31, 8103 (1998).

[43] A. I. Mudrov and K. B. Varnashev, Phys. Rev. B 64, 214423 (2001).

[44] A. I. Sokolov and K. B. Varnashev, Phys. Rev. B 59, 8363 (1999).

[45] A. I. Mudrov and K. B. Varnashev, Phys. Rev. E 58, 5371 (1998).

[46] For comparison, we have also performed the direct analysis of $\omega_{v}$, resumming the expansions for fixed $\bar{w} / \bar{u}$. In zero dimensions, these series are not Borel summable, and this is expected to be true in any dimension. However, for the short series we are considering, we can still hope to obtain reasonable results. We obtain $\omega_{v}=-0.023(20)\{20\}$ (the second error is related to the uncertainty on the coordinates of the fixed point) in agreement with the one given in the text.

[47] We are indebted with Christian Tanguy for informing us of the systematic relation of the coefficients of the series for the cubic model that corresponds to Eq. (A2).

[48] D. Mukamel, Phys. Rev. Lett. 34, 481 (1975).

D. Mukamel and S. Krinsky, J. Phys. C 8, L496 (1975); Phys. Rev. B 13, 5065 (1976). 